\title{
Review of Noncontact Ultrasonic Measurement System for Structural Health Monitoring
}

\author{
Makiko Kobayashi ${ }^{1 *}$, Wu $\mathrm{KT}^{2}$ and Riichi Murayama ${ }^{3}$ \\ ${ }^{1}$ Kumamoto University, Japan \\ ${ }^{2}$ National Research Council of Canada, Canada \\ ${ }^{3}$ Fukuoka Institute of Technology, Japan
}

ISSN: 2639-0574

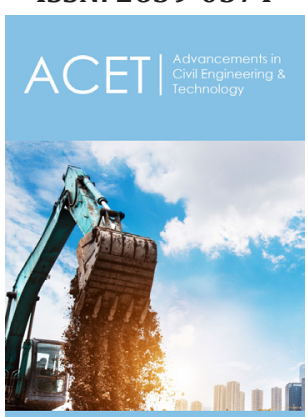

${ }^{* 1}$ Corresponding author: Makiko Kobayashi, Graduate school of Science and Technology, Japan

Submission: 海 September 04, 2019

Published: 覂September 12, 2019

Volume 3 - Issue 4

How to cite this article: Makiko Kobayashi, Wu KT and Riichi Murayama. Review of Noncontact Ultrasonic Measurement System for Structural Health Monitoring. Adv Civil Eng Tech. 3(4). ACET.000569.2019.

DOI: 10.31031/ACET.2019.03.000569

Copyright@ Makiko Kobayashi, This article is distributed under the terms of the Creative Commons Attribution 4.0 International License, which permits unrestricted use and redistribution provided that the original author and source are credited.

\begin{abstract}
Non-destructive testing (NDT) of infrastructure such as bridges and tunnels has become more important since it has aging problem and traffic was heavier than expected. Thin film ultrasonic sensors would be attractive because it is easy to integrate on huge structure. However, NDT of huge structure is still challenging because of sensor accessibility. Semi-noncontact ultrasonic sensors were proposed in this manuscript. $20 \mathrm{~cm}$ diameter copper coil transmit/receive electrical signal from a pulser/receiver instrument and a thin film ultrasonic sensor and reflected echo from $12.7 \mathrm{~mm}$ thick steel plate successfully. By this method, measurement using drone could be possible and it increases application possibility for huge infrastructure monitoring.
\end{abstract}

Keywords: NDT, Non-contact sensing; Ultrasound

\section{Introduction}

Non-destructive testing (NDT) of infrastructures, such as bridges and tunnels, is important to maintain the civil infrastructures [1,2]. It has become more important since it has aging problem and traffic load was much heavier than calculated values which were derived several decays years ago. However, even though it is very important to monitor civil infrastructures, it is very difficult to carry out quantitative evaluation by traditional NDT. Since structures are huge and high, once the structures were assembled, it is very hard to reach inspection points. Recently, drones are applied for many applications, and structural health monitoring is promising applications since for drones it is easy to approach the structures with height $[3,4]$. If drones can attach the wall of civil infrastructure, it is possible to use traditional ultrasonic sensors which can measure internal conditions [3], however, to attach the wall, very matured control technique of drones are required, so non-contact ultrasonic method is better. There are three traditional non-contact ultrasonic method. First one is air-coupled ultrasonic transducers.

Recently, air-coupled ultrasonic transducers made by Capacitive Micromachined Ultrasonic Transducer (CMUT) has been developed and the transmitting powder and the resolution has been improved [5]. However, air-coupled ultrasonic transducers are often used to detect obstacles in the air, and it is still challenging to inspect inside of the structures due to acoustic impedance difference. Incident angle is also critical. Electromagnetic acoustic transducers (EMATs) are applied high temperature metallic structures [6]. Intrinsically, this technique can be applied for only conductive material so that it is not suitable for many civil infrastructure components. In laser ultrasound, laser induced ultrasound, i.e., inspection structure itself is ultrasound transmitter and reflected ultrasound is picked up by optical method [7]. This technique is suitable to detect huge and complex geometry and there is no incident angle problem. already applied mainly in aerospace industry. However, since ultrasound generation efficiency is low so that optical detection system is costly. To carry out ultrasonic measurement without wires (kind of non-contact) in relatively low cost for civil infrastructures, combination of thin film ultrasonic sensors and wireless power transmission was tested.

\section{Experimental method}

A thin film ultrasonic sensor was fabricated on $12.7 \mathrm{~mm}$ thick steel substrate by sol-gel spray method. Thin film ultrasonic sensors made by sol-gel composite materials have been 
developed for high temperature applications. If thin films fabricated on flexible substrate, thin film flexible ultrasonic sensors can be realized, and the sensor performance could be comparable with commercial ultrasonic transducers [8]. One of the advantages of thin film ultrasonic sensors is facility of huge structure integration as shown in Figure 1. For room temperature applications, there is no requirement of heat treatment for infrastructures so that there is no concern of thermal damage and less time consuming for sensor integration. In this experiment, lead zirconate titanate (PZT) powders and PZT sol-gel solution were used to synthesize PZT/PZT composite. Mixture of PZT powders and PZT sol-gel was sprayed on the steel block.

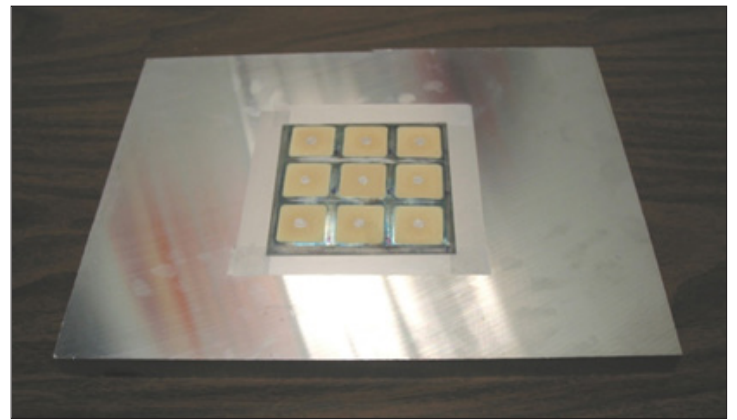

Figure 1: Thin film sensors fabricated on $10 \mathrm{~cm} \times 10 \mathrm{~cm} \times 75 \mu \mathrm{m}$ stainless steel film mounted on $\sim 20 \mathrm{~cm} \times \sim 30 \mathrm{~cm} \times 1.9 \mathrm{~cm}$ aluminum plate.

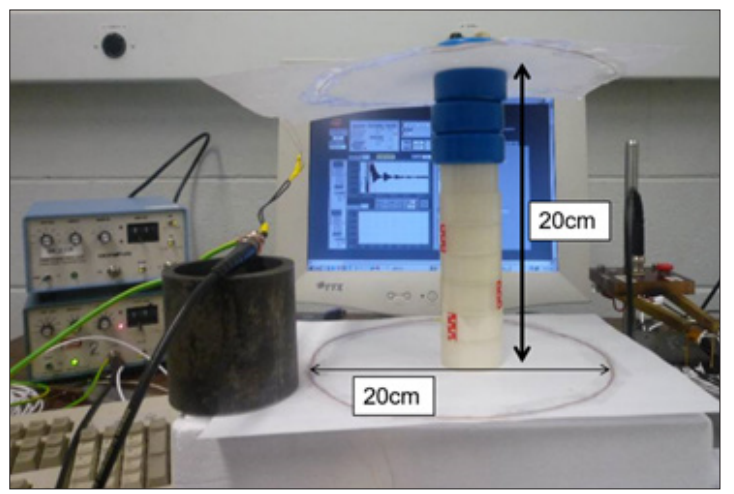

Figure 2: Experimental setup for semi-noncontact ultrasonic measurement when liftoff distance was $20 \mathrm{~cm}$.

After spray coating, thermal treatment was operated by an induction heating machine. Spray coating processes and thermal processes were repeated until the thin film reached the desired thickness for ultrasonic measurement. Ultrasonic sensor fabrication was accomplished after poling and top electrode fabrication. In pulse-echo mode, a pulser/receiver machine (P/R) supplied pulse voltage to piezoelectric thin film via electrodes, then piezoelectric thin film converts voltage to ultrasound. Ultrasound reflected at the bottom surface of the steel black then thin film converted returned ultrasound to electrical signal. Usually, P/R and thin film sensor are electrically connected by electrical cables. However, this electrical connection could be wireless by contactless power transmission [9]. Measurement setup is shown in Figure 2. The two ends of one copper coil with $\sim 20 \mathrm{~cm}$ diameter was connected to the top electrode of thin film ultrasonic sensor. It is noted that the steel substrate serving as the bottom electrode. Another coil was connected to $\mathrm{P} / \mathrm{R}$. Then electrical power was transferred over short distances by electromagnetic induction between $0-20 \mathrm{~cm}$ between two copper coils as shown in Figure 2.

\section{Results and Discussions}

Typical examples of the received signals reflected from the bottom of the $12.7 \mathrm{~mm}$-thick steel substrate in Figure 3 (liftoff distance: $0 \mathrm{~cm}$ ) and Figure 4 (liftoff distance: $20 \mathrm{~cm}$ ), respectively. Time delay $\mathrm{Td}$ of reflected echo could be calculated as following equation;

$$
T_{d}=\frac{2 t}{v}
$$

where $\mathrm{t}$ and $\mathrm{v}$ are thickness and longitudinal velocity of the steel substrate, respectively. Since t was $12.7 \mathrm{~mm}$ and $\mathrm{v}$ was material constant. Since the steel substrate material was AISI P20 steel so that $\mathrm{v}$ was estimated as $\sim 5900 \mathrm{~m} / \mathrm{s}$. Therefore, according to the above-mentioned equation, Td was calculated as $\sim 4.3 \mu \mathrm{s}$ and it was reasonably agreed with periodical electrical signal peak time difference after $\sim 5 \mu \mathrm{s}$ in Figure $3 \& 4$. It seems that electrical power transmission for ultrasonic measurement in pulse-echo mode was successfully operated with $20 \mathrm{~cm}$ distance. As liftoff distance increased, signal quality decreased. It could be solved by using alternative wireless power transmission method, such as resonant inductive coupling. Further research is required for semi-contact ultrasonic measurements using drones.

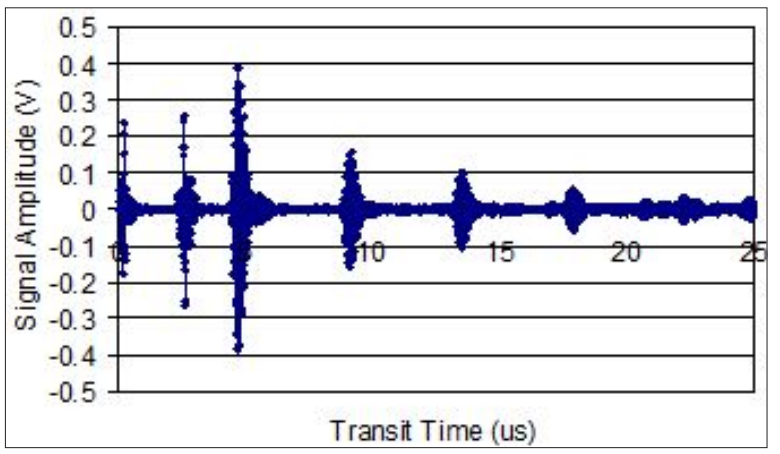

Figure 3: Examples of ultrasonic measurement result when liftoff $=0 \mathrm{~cm}$.

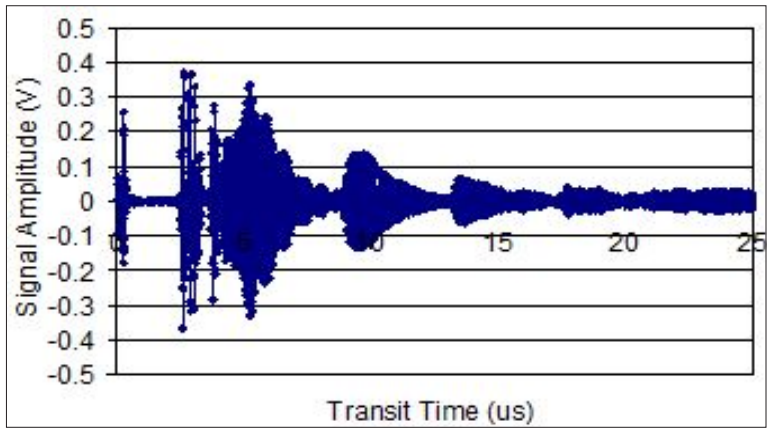

Figure 4: Examples of ultrasonic measurement result when liftoff $=20 \mathrm{~cm}$. 


\section{References}

1. Sanjeev Kumar V, Sudhir Singh B, Saleem A (2013) Review of nondestructive testing methods for condition monitoring of concrete structures. J Constr Eng.

2. Hoshyar AN, Maria Rashidi, Ranjith L, Bijan Samali (2019) Algorithm development for the non-destructive testing of structural damage. Appl Sci 2810.

3. Rami AM, Remy Kalai (2018) Development of a wall-sticking drone for non-destructive ultrasonic and corrosion testing. Drones 2(1): 8.

4. Carla Alves M, De Souza C, Tsukasa M, da Silva AG (2012) In-service flares inspection by unmanned aerial vehicles (UAVs). Proc. $18^{\text {th }}$ World Conference on Nondestructive Testing

5. Shuai Na, Zhou Zheng, Hsiang Chen AI, Lawrence LPW, Zhenhao Li, et al. (2017) Design and fabrication of a high-power air-coupled capacitive micromachined ultrasonic transducer array with concentric annular cells. IEEE Trans Electr Devices 64: 4636-4643.

6. Pei C, Xiao P, Zhao S, Chen Z, Takagi T (2017) A flexible film type EMAT for inspection of small diameter pipe. J Mech Sci Technol 31(8): 36413645 .

7. PétillonJ O, Dupuis P, David D, Voillaume H, Trétout H (1995) Laser ultrasonics: A non-contacting NDT system. Review Progr Quant Nondestr Eval, pp. 1189-1195.

8. Kobayashi M, Jen CK, Lévesque D (2004) Flexible ultrasonic transducers. IEEE Trans. Ultrason Ferroelect Freq Contr 53(8): 1478-1486.

9. Murayama R, Kobayashi M, Wu KT, Jen CK (2012) Noncontact driving system using induction-based method and integrated piezoelectric ultrasonic transducers. J Sensor Technol 2: 60-67.

For possible submissions Click below: 